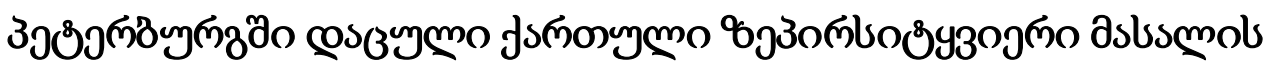

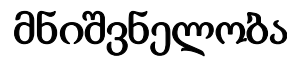

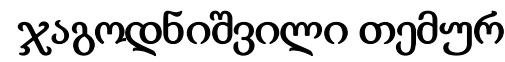

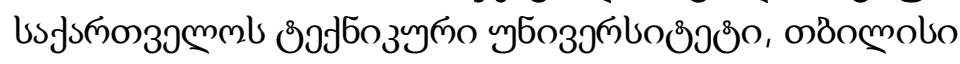 \\ https://doi.org/10.52340/idw.2021.526
}

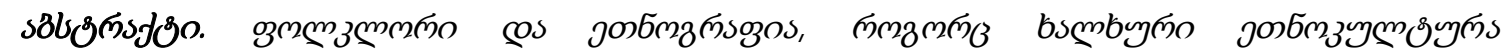

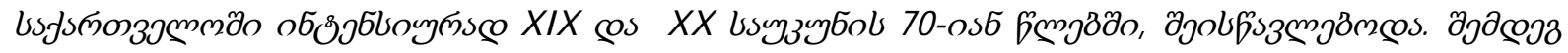

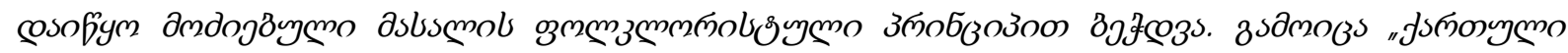

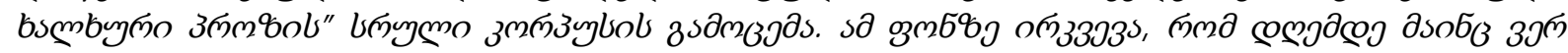

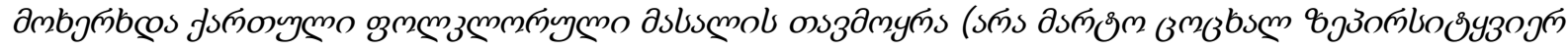

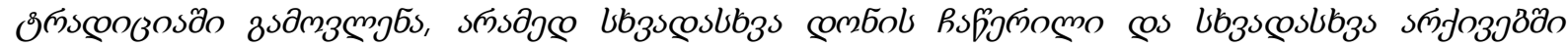

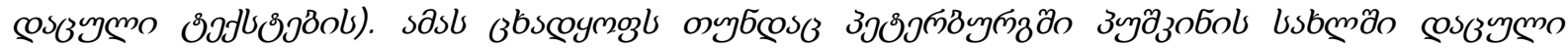

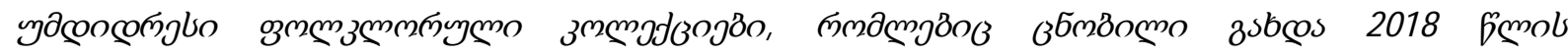

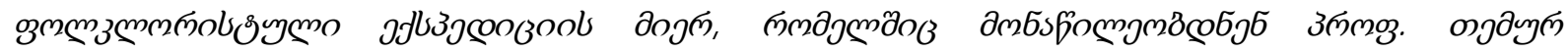

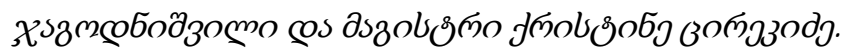

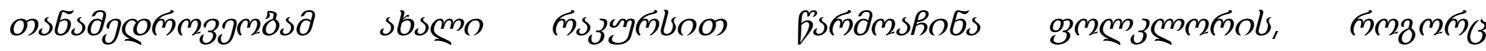

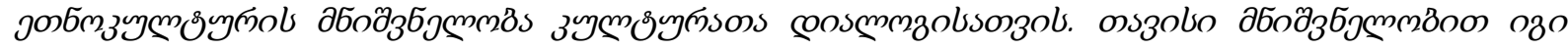

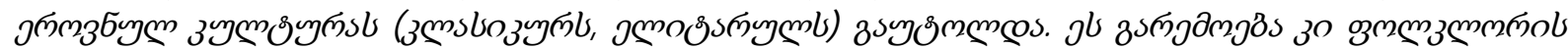

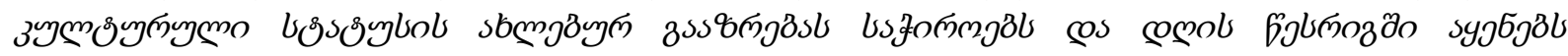

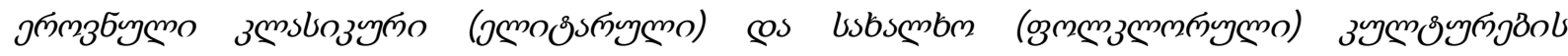
coosmmzol us fonmast.
\end{abstract}

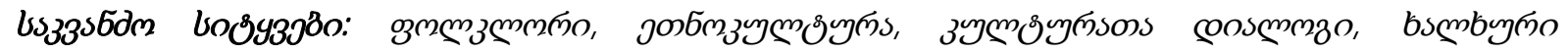

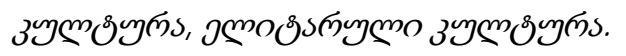

\section{1. ajuszsmo}

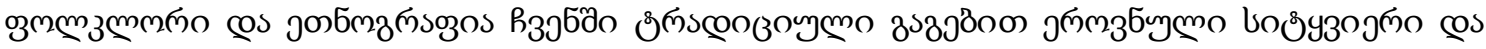

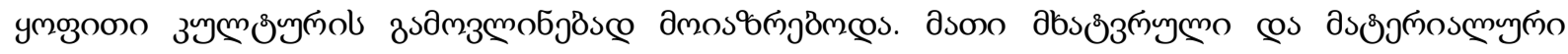

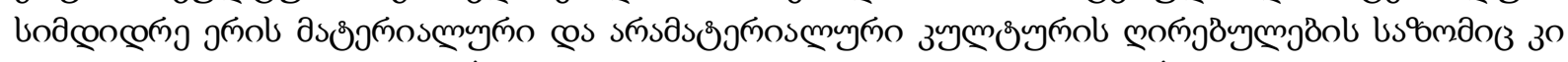

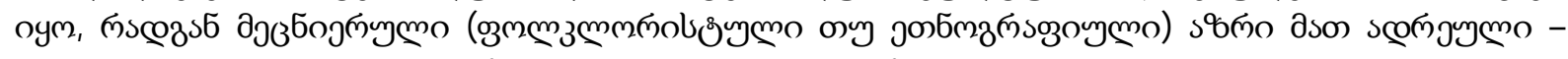

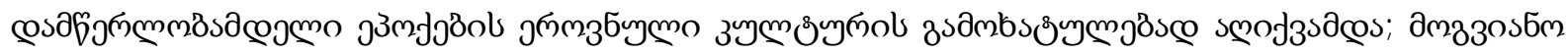

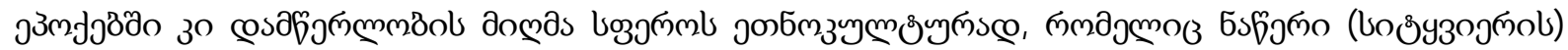

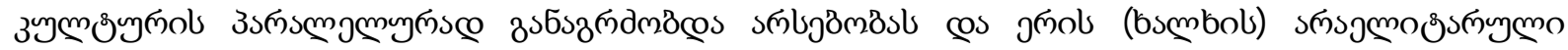

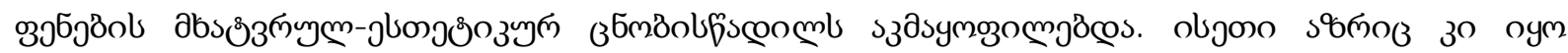

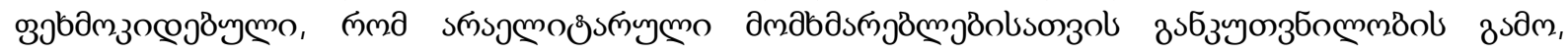

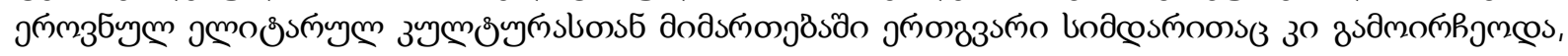

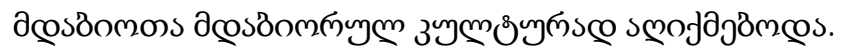

\section{2. domoosscon 6sfomo}

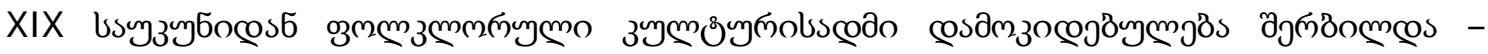

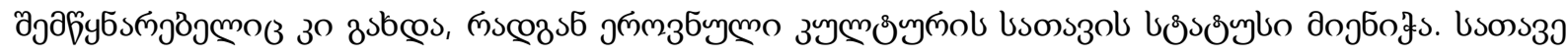

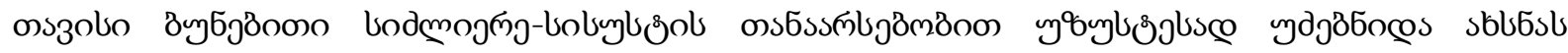

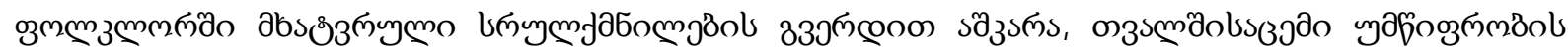




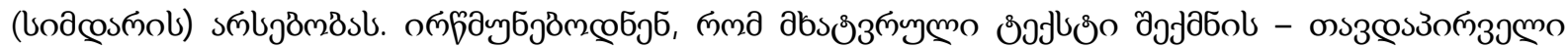

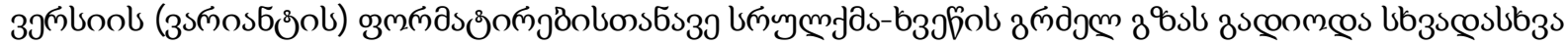

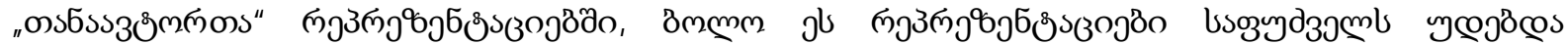

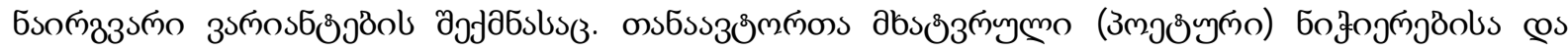

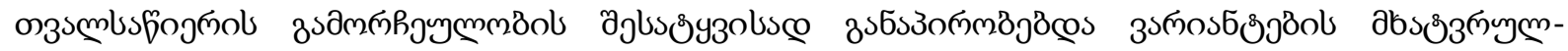

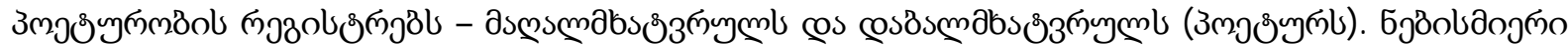

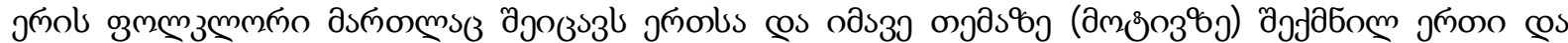

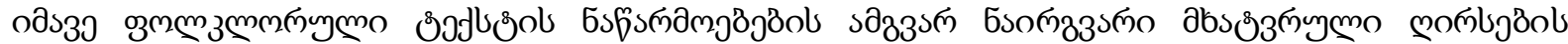
з ґпоббీ

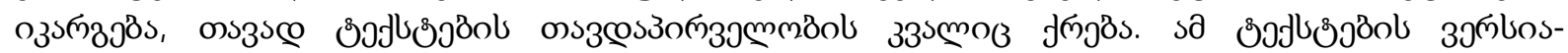

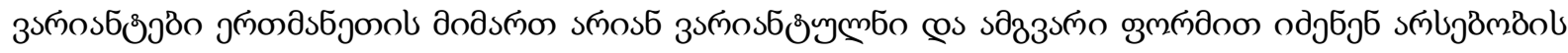

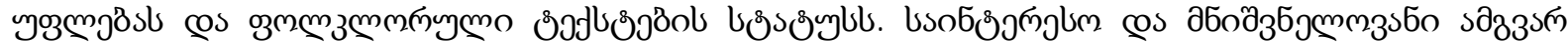

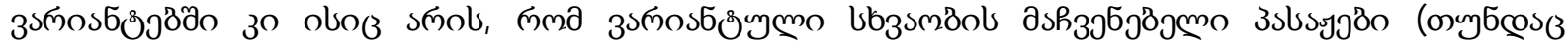

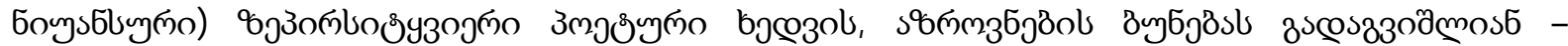

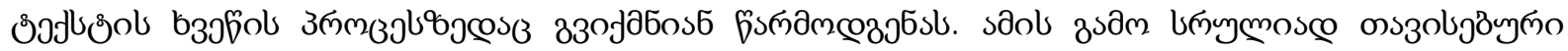

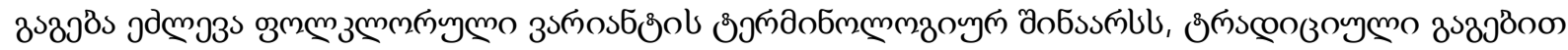

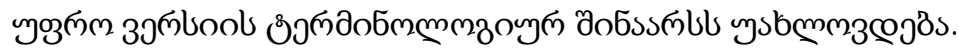

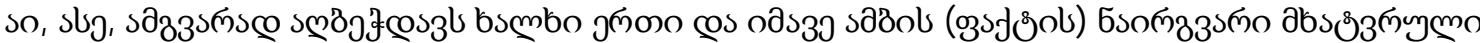

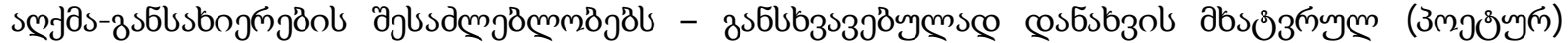

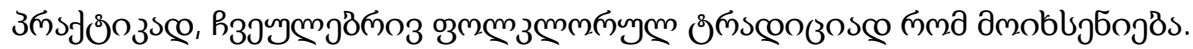

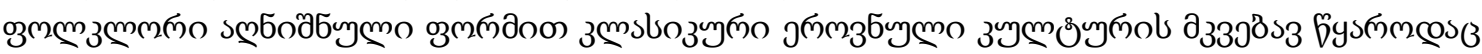

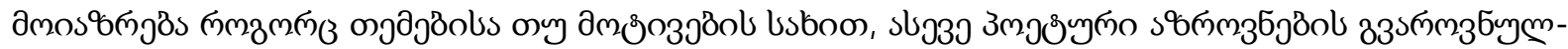

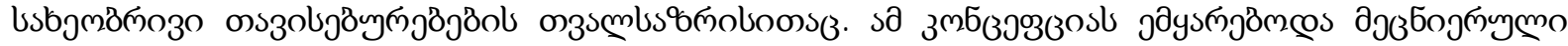

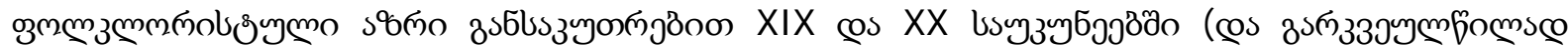

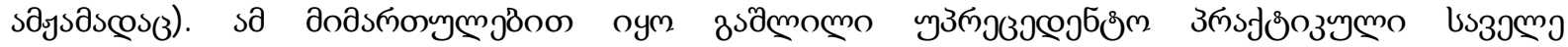

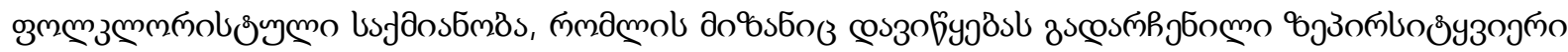

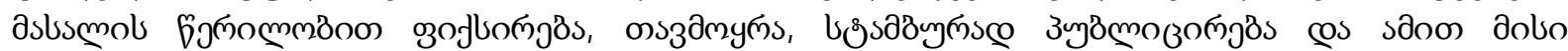

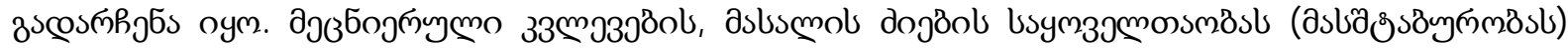

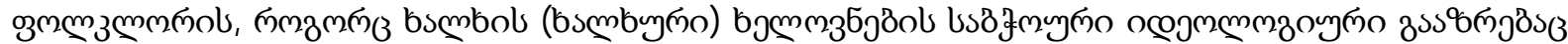

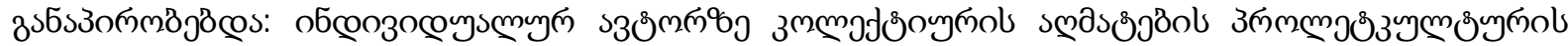
"Oुommlimos".

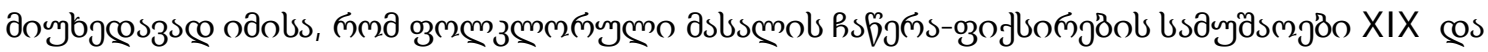

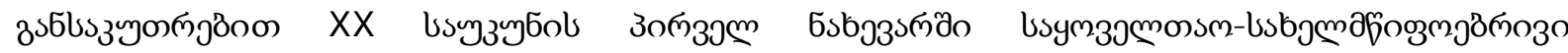

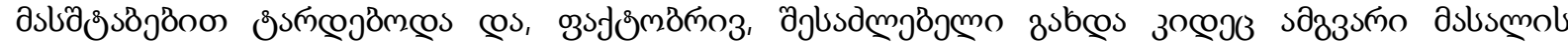
yansz

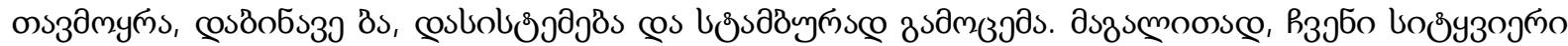

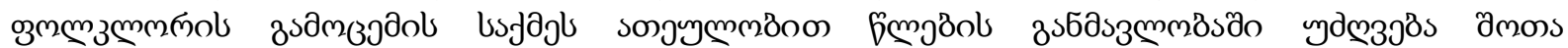

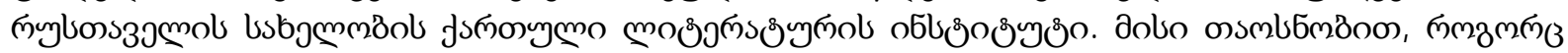

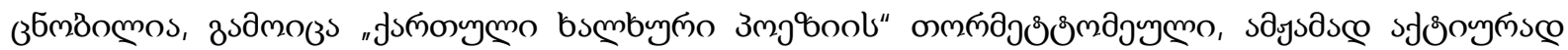

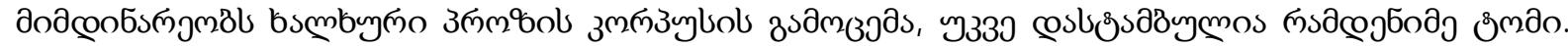

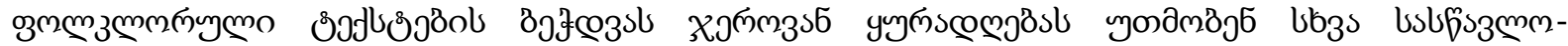

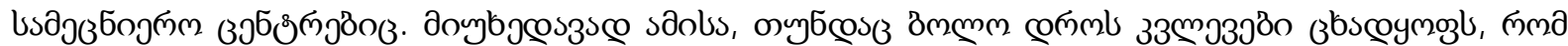

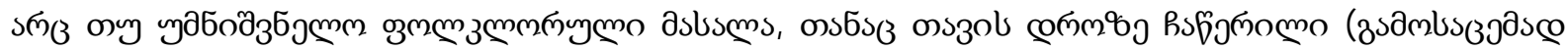

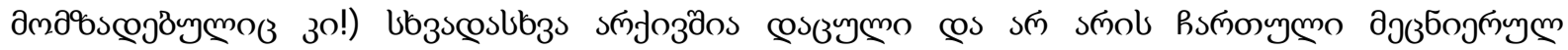

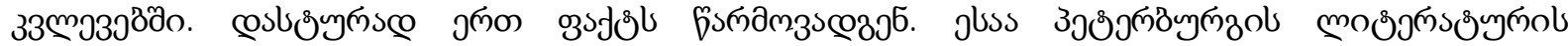

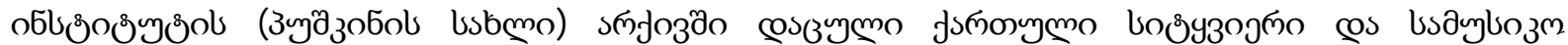

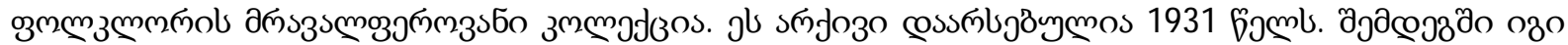

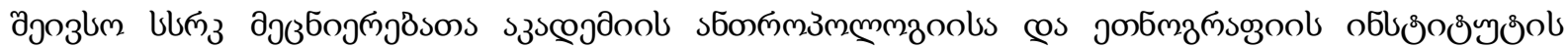

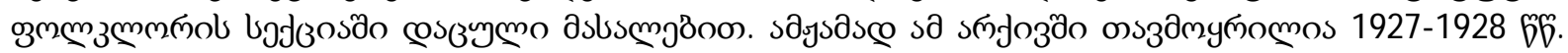

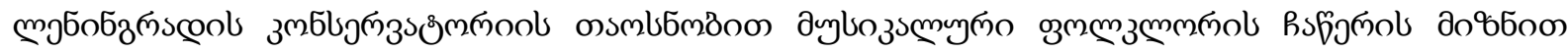




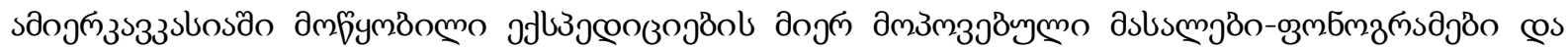

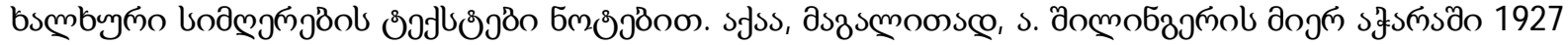

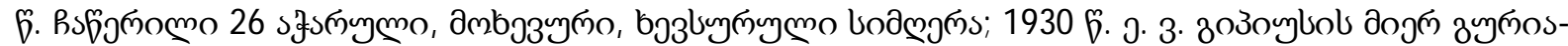

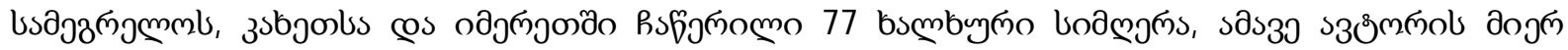

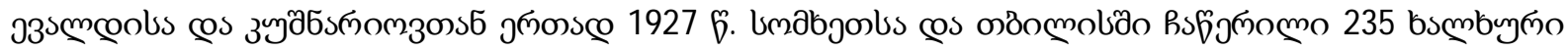

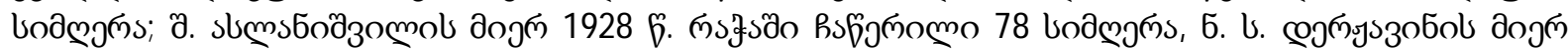

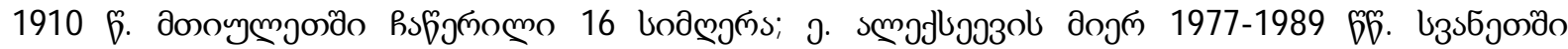

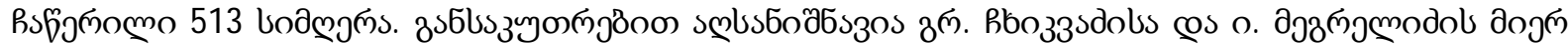

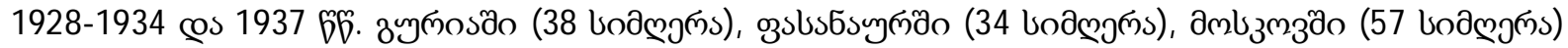

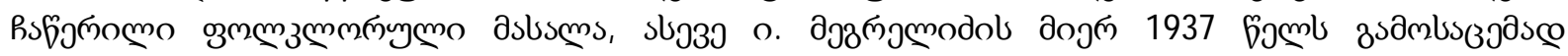

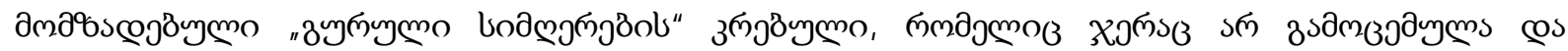

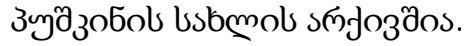

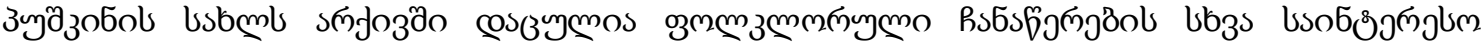

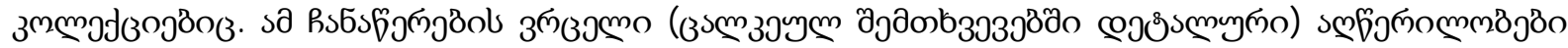
fiпдм@

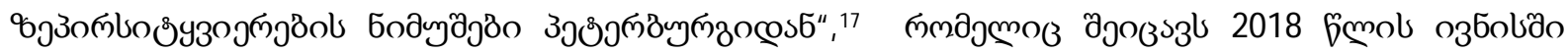

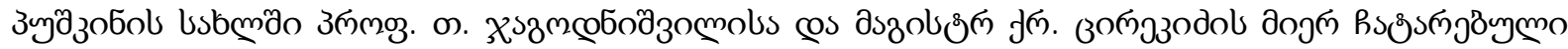

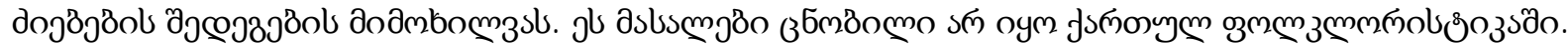

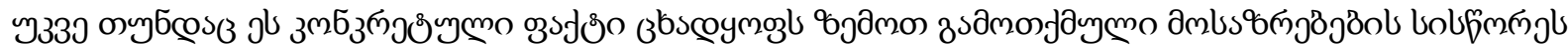

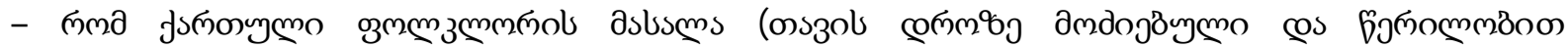

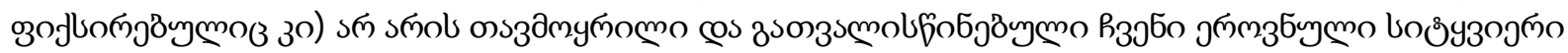

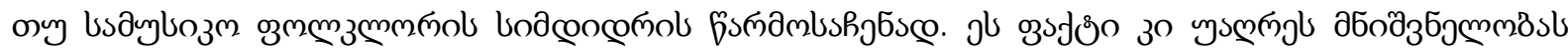

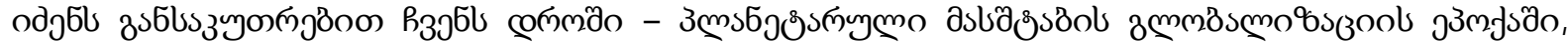

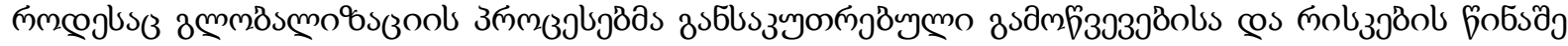

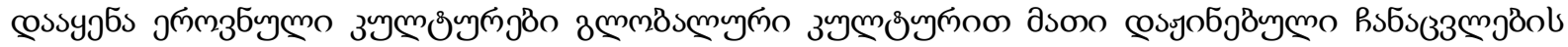

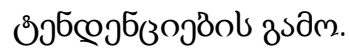

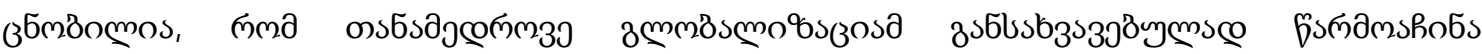

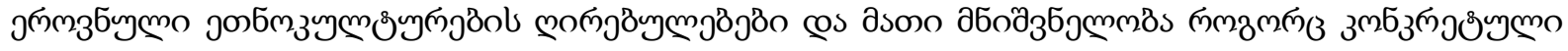

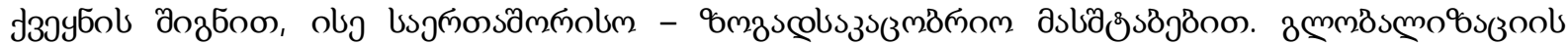

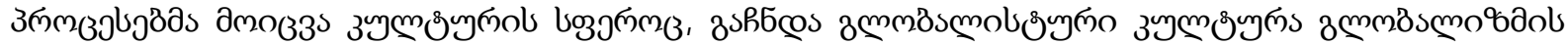

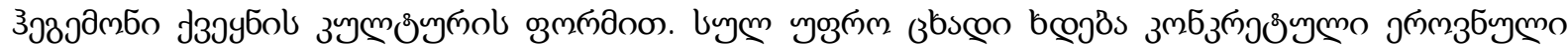

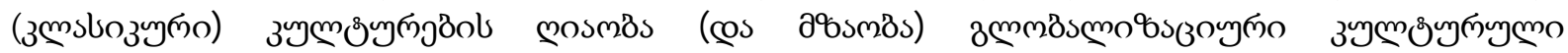

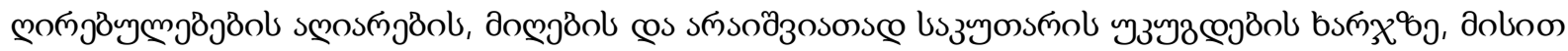

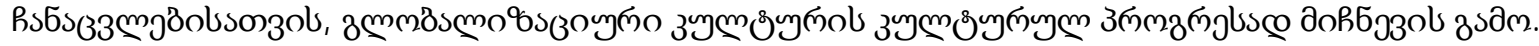

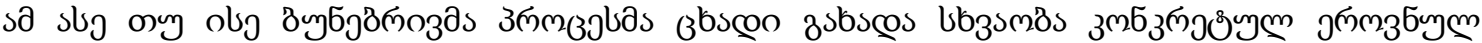

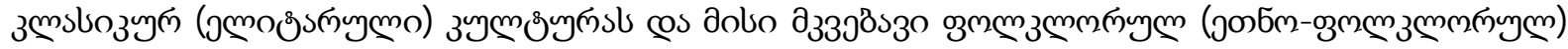

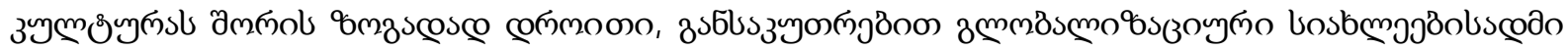

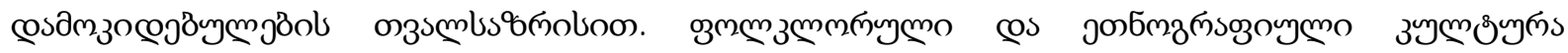

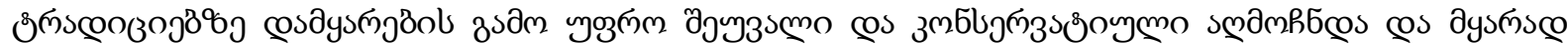

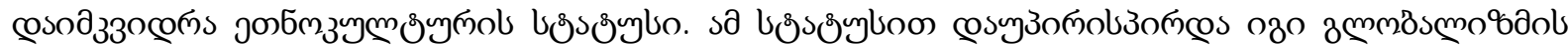

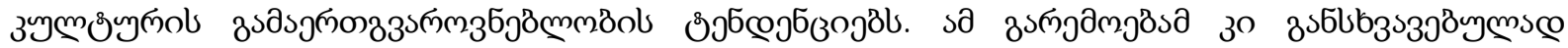

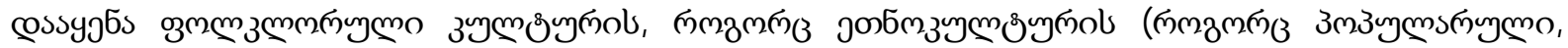

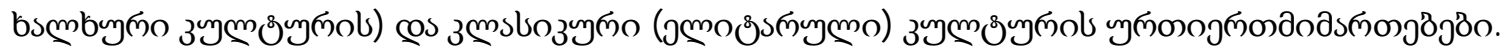

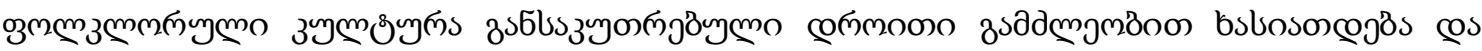

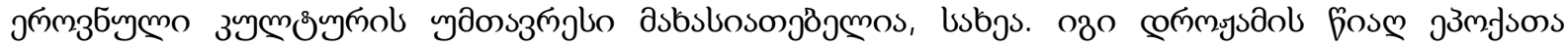

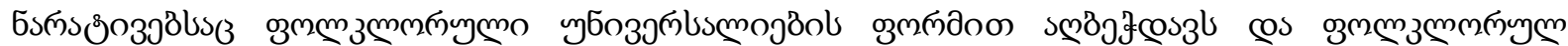

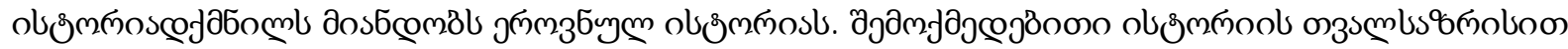

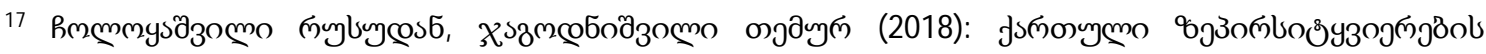

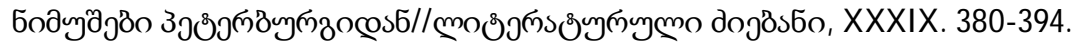




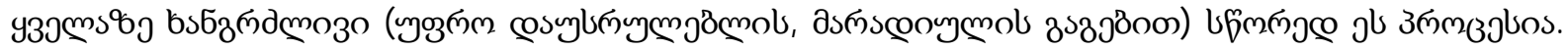

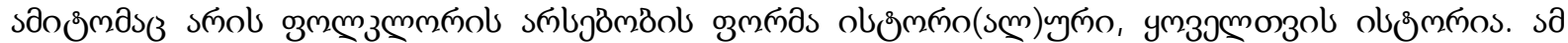

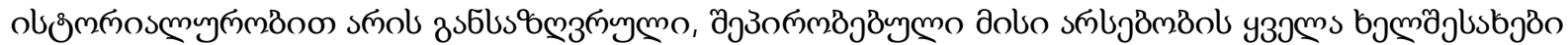

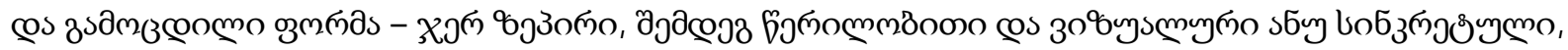

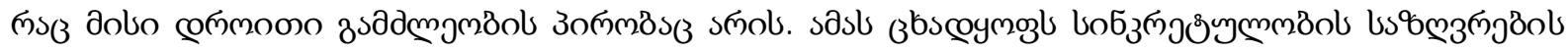

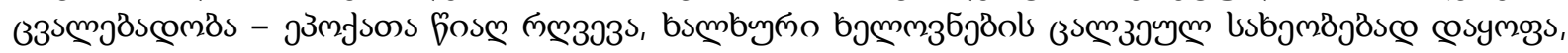

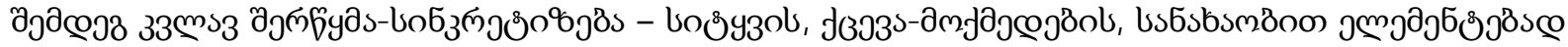

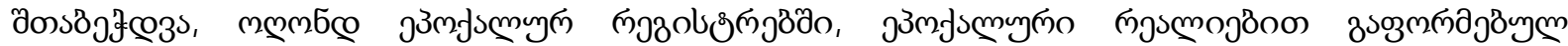

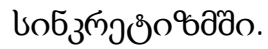

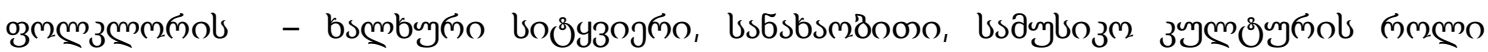

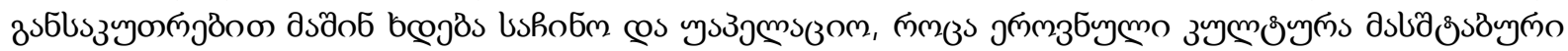

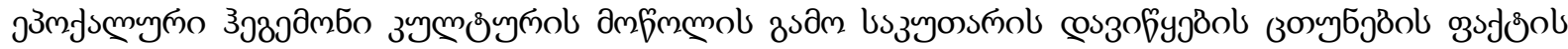

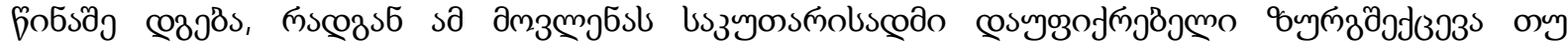

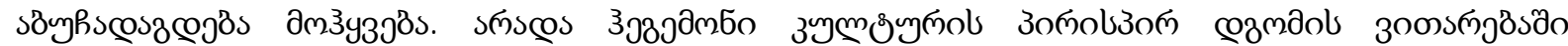

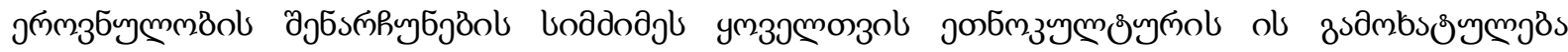

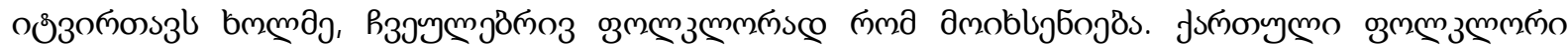

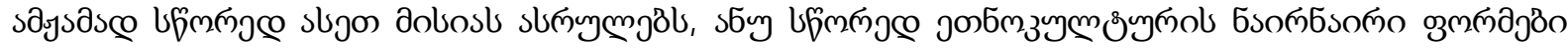

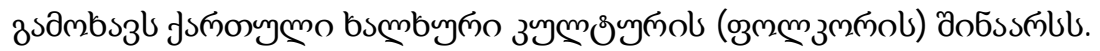

\section{3. coslis36s}

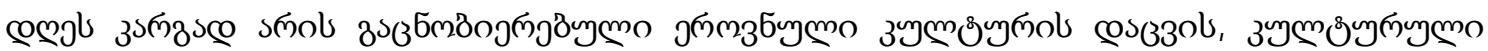

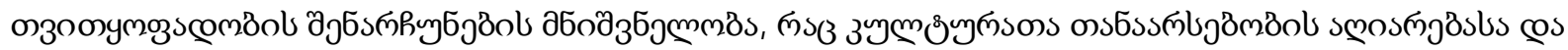

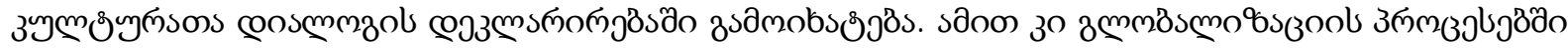

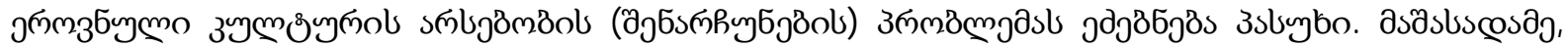

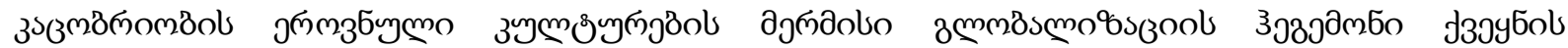

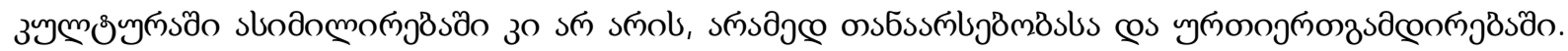

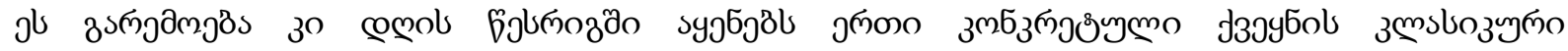

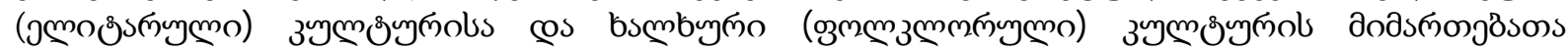

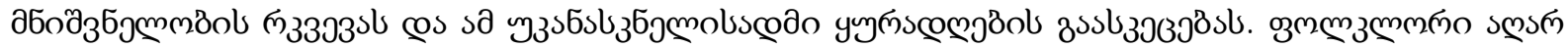

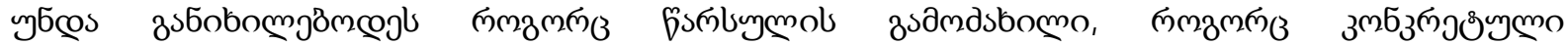

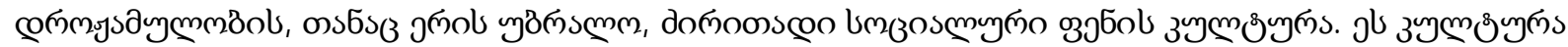

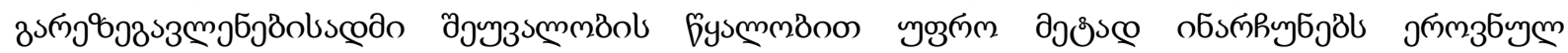

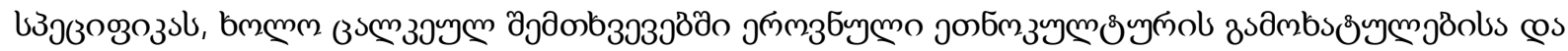

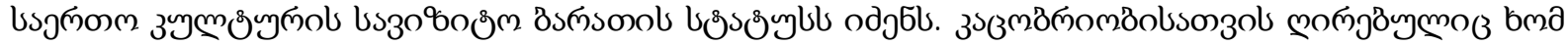

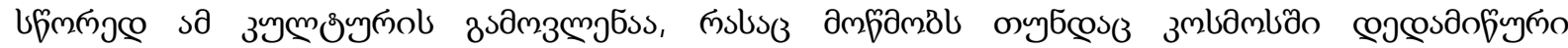

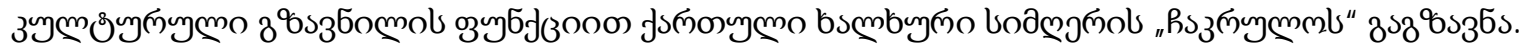

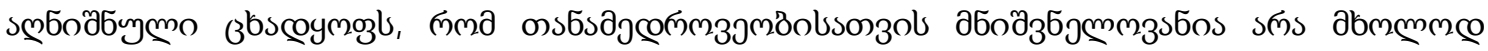

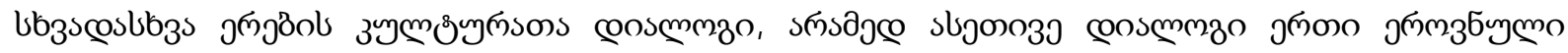

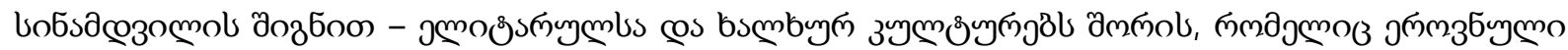

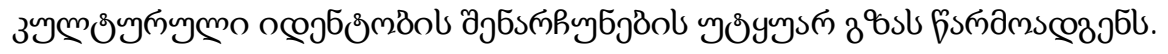




\title{
The Importance of Georgian Oral Material Preserved in Saint Petersburg
}

Jagodnishvili Temur

Georgian Technical University, Tbilisi

\begin{abstract}
Folklore and ethnography as a folk ethnoculture were intensively studied in Georgia in the 70s of the XIX and XX centuries. Then the found material has been printed according to the folkloric principle. The publication of the complete corpus of Georgian folk prose was published. On this background, it is clear that Georgian folklore has not been collected so far (nor in the living oral tradition, neither in texts recorded at different levels and preserved in various archives). This is evidenced even by the richest folklore collections preserved at the Pushkin House in St. Petersburg, which became known by the folklore expedition in 2018 .

Modernity has presented a new perspective on the importance of folklore as an ethnoculture for the dialogue of cultures. In its significance, it has been equated with national culture (classical, elitist). This circumstance requires a new understanding of the cultural status of folklore and puts on the agenda of necessity for dialogue between national classical (elite) and folk (folk) cultures.
\end{abstract}

Key words: Folklore, ethnocultural, dialogue of cultures, folk culture, elite culture. 